\title{
Supporting Online Material for The PROFOUND database for evaluating vegetation models and simulating climate impacts on forests
}

Christopher P.O. Reyer ${ }^{1}$, Ramiro Silveyra Gonzalez ${ }^{1}$, Klara Dolos ${ }^{3}$, Florian Hartig ${ }^{4}$, Ylva Hauf $^{1}$, Matthias Noack ${ }^{5}$, Petra Lasch-Born ${ }^{1}$, Thomas Rötzer ${ }^{6}$, Hans Pretzsch ${ }^{6}$, Henning Meesenburg ${ }^{7}$, Stefan Fleck ${ }^{7}$, Markus Wagner ${ }^{7}$, Andreas Bolte ${ }^{8}$, Tanja GM Sanders ${ }^{8}$, Pasi Kolari $^{9}$, Annikki Mäkelä ${ }^{9}$, Timo Vesala ${ }^{9}$, Ivan Mammarella ${ }^{9}$ Jukka Pumpanen ${ }^{10}$, Alessio Collalti $^{11}{ }^{12}$, Carlo Trotta ${ }^{12}$, Giorgio Matteucci ${ }^{13}$, Ettore D’Andrea ${ }^{13}$, Lenka Foltýnová ${ }^{14}$, Jan Krejza $^{14}$, Andreas Ibrom ${ }^{15}$, Kim Pilegaard ${ }^{15}$, Denis Loustau ${ }^{16}$, Jean-Marc Bonnefond ${ }^{16}$, Paul Berbigier $^{16}$, Delphine Picart ${ }^{16}$, Sébastien Lafont ${ }^{16}$, Michael Dietze ${ }^{17}$, David Cameron ${ }^{18}$, Massimo Vieno ${ }^{19}$, Hanqin Tian ${ }^{20}$, Alicia Palacios-Orueta ${ }^{21}$, Victor Cicuendez ${ }^{21}$, Laura Recuero $^{21}$, Klaus Wiese ${ }^{21}$, Matthias Büchner ${ }^{1}$, Stefan Lange ${ }^{1}$, Jan Volkholz ${ }^{1}$, Hyungjun $\mathrm{Kim}^{22}$, Graham P. Weedon ${ }^{27}$, Justin Sheffield ${ }^{28}$, Iliusi Vega del Valle ${ }^{1}$, Felicitas Suckow ${ }^{1}$, Joanna A. Horemans ${ }^{23}$, Simon Martel $^{16}$, Friedrich Bohn ${ }^{24}$, Jörg Steinkamp ${ }^{25}$, Alexander Chikalanov $^{26}$, Mats Mahnken ${ }^{1}$, Martin Gutsch ${ }^{1}$, Katja Frieler ${ }^{1}$

${ }^{1}$ Potsdam Institute for Climate Impact Research, Member of the Leibniz Association, P.O.

Box 601203, D-14412 Potsdam, Germany

${ }^{2}$ University of Freiburg, Freiburg, Germany

${ }^{3}$ Karlsruhe Institute of Technology (KIT), Karlsruhe, Germany

${ }^{4}$ University of Regensburg, Regensburg, Germany

${ }^{5}$ Fachagentur Nachwachsende Rohstoffe e.V. (FNR), Gülzow-Prüzen, Germany

${ }^{6}$ Technical University of Munich, Munich, Germany

${ }^{7}$ Northwest German Forest Research Institute, Göttingen, Germany

${ }^{8}$ Thünen Institute of Forest Ecosystems, 16225 Eberswalde, Germany

${ }^{9}$ University of Helsinki, Helsinki, Finland

${ }^{10}$ University of Eastern Finland, Kuopio, Finland

${ }^{11}$ National Research Council of Italy, Institute for Agriculture and Forestry Systems in the Mediterranean, Rende (CS), Italy

${ }^{12}$ Department of Innovation in Biological, Agro-food and Forest System, University of Tuscia, 01100 Viterbo, Italy

${ }^{13}$ National Research Council of Italy, Institute for Agriculture and Forestry System in the Mediterranean, Ercolano (NA), Italy

${ }^{14}$ Global Change Research Institute, Brno, Czech Republic

${ }^{15}$ Technical University of Denmark, Lyngby, Denmark

${ }^{16}$ French National Institute for Agricultural Research, Bordeaux, France

${ }^{17}$ Boston University, Boston, USA

${ }^{18}$ Centre for Ecology and Hydrology, Lancaster, United Kingdom

${ }^{19}$ Centre for Ecology and Hydrology, Edinburgh, United Kingdom

${ }^{20}$ Auburn University, Auburn, United States

${ }^{21}$ Technical University of Madrid, Madrid, Spain

${ }^{22}$ University of Tokyo, Tokyo, Japan

${ }^{23}$ Centre of Excellence PLECO, University of Antwerpen, Antwerpen, Belgium

${ }^{24} \mathrm{Helmholz}$ Center for Environmental Research, Leipzig, Germany

${ }^{25}$ Senckenberg Biodiversity and Climate Research Centre, Senckenberg, Germany

${ }^{26}$ University of Library Study and Information Technology, Sofia, Bulgaria

${ }^{27}$ Met Office, Wallingford, UK

${ }^{28}$ Princeton University, Dept. Civil \& Environ. Eng., Princeton, NJ 08544, USA

Correspondence to: Christopher P.O. Reyer (reyer@pik-potsdam.de) 

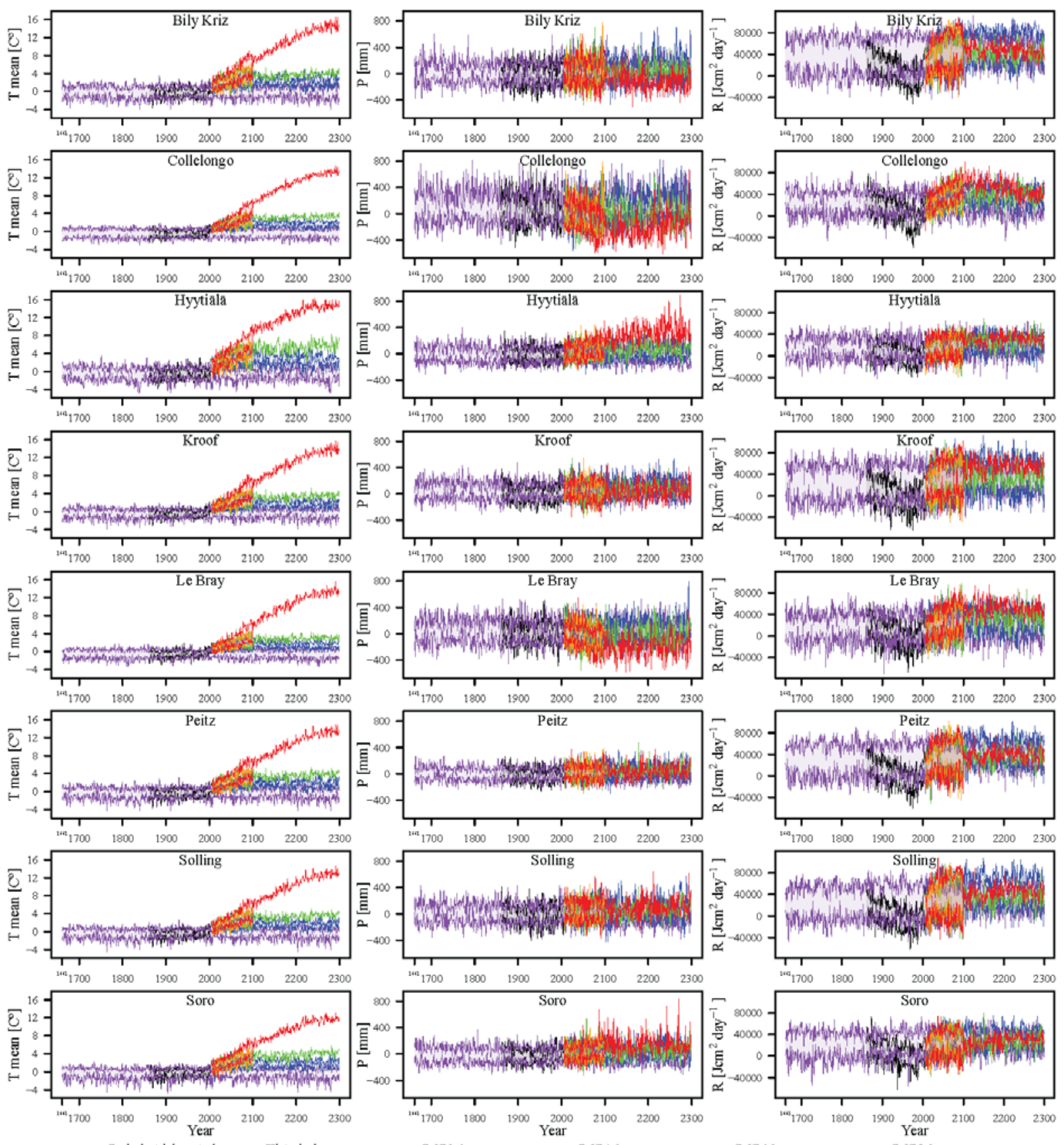

Figure SOM1: Change in mean annual temperature ( $T$ mean), annual precipitation sum (P) and annual sum of global radiation (R) over the time period 1661-2299 relative to the 19802005 average for the ISIMIP2b scenarios. Please note that the two Solling sites have the same climate. 

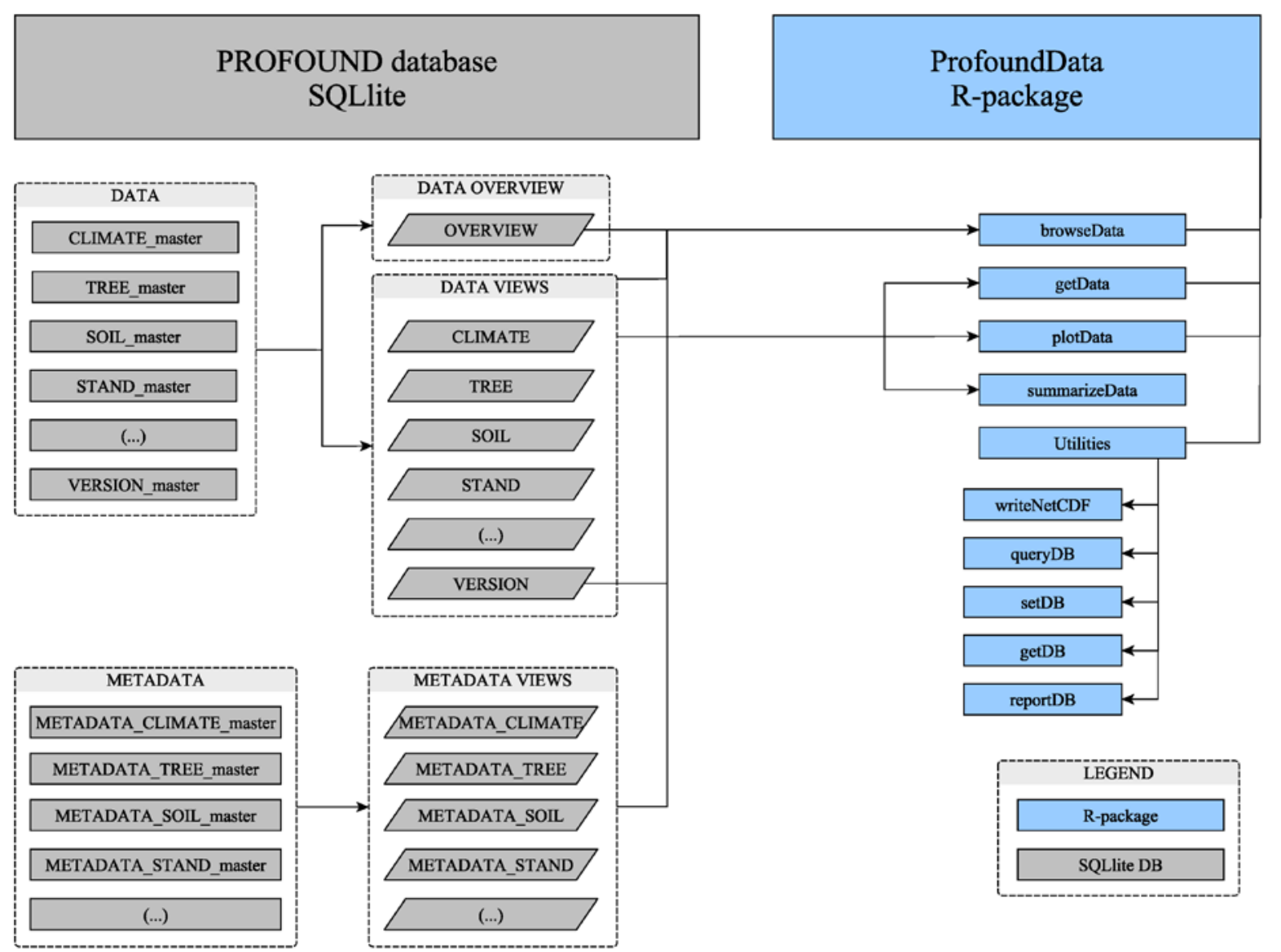

Figure SOM2: The main structure and links between the PROFOUND DB (grey) and the PROFOUNDData R-package (blue). 

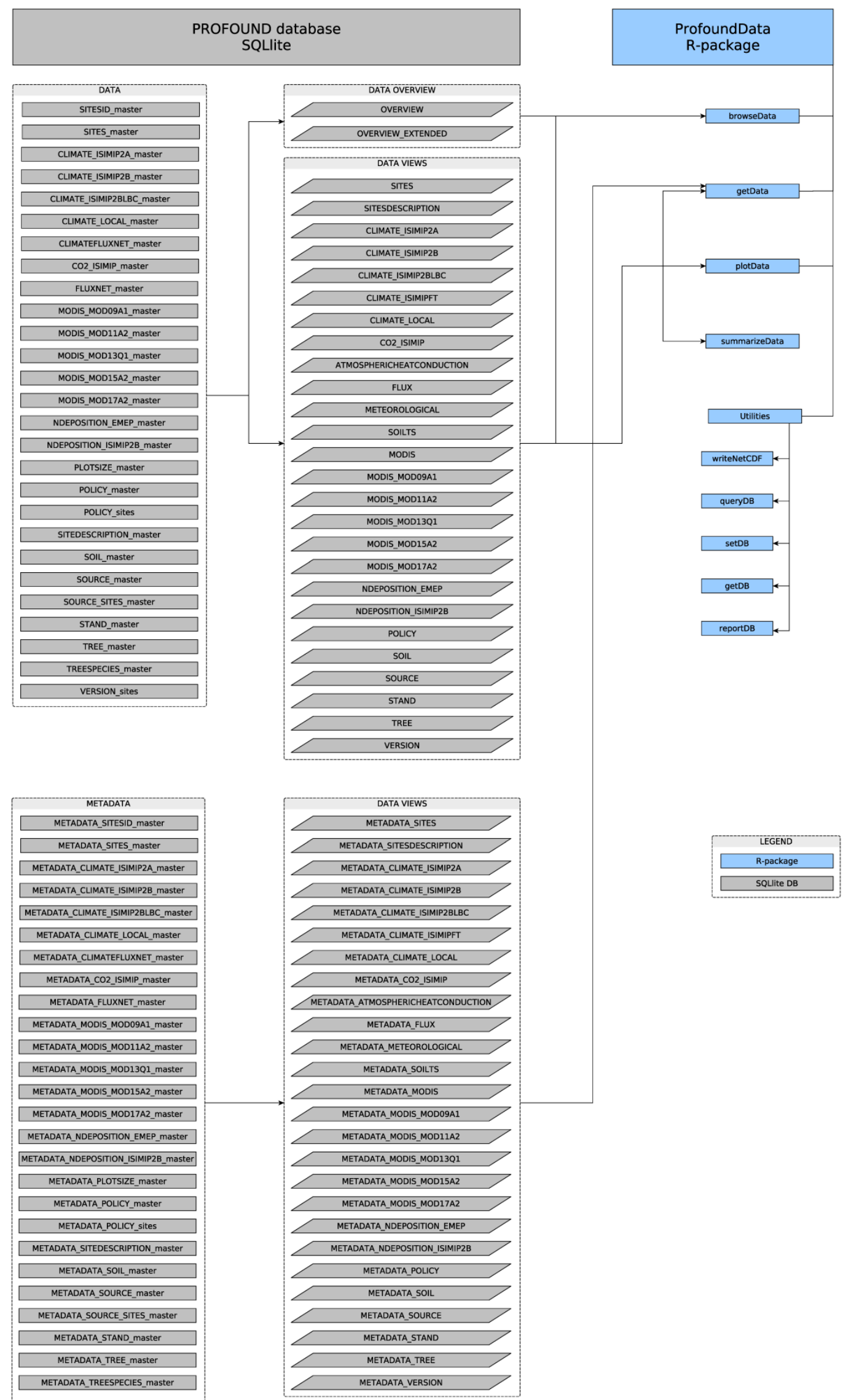

Figure SOM3: The full structure and links between the PROFOUND DB (grey) and the PROFOUNDData R-package (blue). 
Table SOM1: Description of SITES variables included in the database.

\begin{tabular}{|l|l|l|l|}
\hline variable & type & units & description \\
\hline site & TEXT & adimensional & Site name \\
\hline site_id & $\begin{array}{l}\text { INTEGE } \\
\text { R }\end{array}$ & adimensional & Site code as decimal number (01-99) \\
\hline aspect_deg & REAL & degree & Direction of slope inclination. Degrees against North. No Value indicates no exposition. \\
\hline country & TEXT & adimensional & Country \\
\hline elevation_masl & REAL & m & Elevation above sea level as recorded by PI \\
\hline epsg & $\begin{array}{l}\text { INTEGE } \\
\text { R }\end{array}$ & adimensional & EPSG Coordinate System \\
\hline lat & REAL & degree decimal & Latitude \\
\hline lon & REAL & degree decimal & Longitude \\
\hline natVegetation_code1 & TEXT & adimensional & $\begin{array}{l}\text { Code of the vegetation mapping unit group in thel"Map of the Natural Vegetation of Europe". } \\
\text { Massstab 1:2.500.000 Karten und Legende. Teil 1-3.. Bundesamt fuer Naturschutz, Bonn, } \\
\text { Germany. }\end{array}$ \\
\hline natVegetation_code2 & TEXT & adimensional & $\begin{array}{l}\text { Code of the vegetation mapping unit in the "Map of the Natural Vegetation of Europe". BOHN, U.; } \\
\text { GOLLUB, G. \& HETTWER, C. (2000) Karte der natuerlichen Vegetation Europas. Massstab } \\
1: 2.500 .000 \text { Karten und Legende. Teil 1-3.. Bundesamt fuer Naturschutz, Bonn, Germany. }\end{array}$ \\
\hline natVegetation_descriptio \\
n
\end{tabular}


Table SOM2: Description of SOIL variables included in the database.

\begin{tabular}{|c|c|c|c|}
\hline variable & type & units & description \\
\hline record_id & $\begin{array}{l}\text { INTEGE } \\
\text { R }\end{array}$ & adimensional & Record ID as decimal number \\
\hline site & TEXT & adimensional & Site name \\
\hline site_id & $\begin{array}{l}\text { INTEGE } \\
\text { R }\end{array}$ & adimensional & Site code as decimal number (1-99) \\
\hline date & TEXT & adimensional & $\begin{array}{l}\text { Unformatted date of inventory as provided for the inventory. See site specific metadata for further } \\
\text { information on date. }\end{array}$ \\
\hline bs_percent & REAL & percent & Percentage of alkaline and earth alkaline metals at CEC \\
\hline cMax_percent & REAL & percent & Maximum soil carbon content \\
\hline cMin_percent & REAL & percent & Minimum soil carbon content \\
\hline cOrgSigma_percent & REAL & percent & Soil organic carbon content error estimate as standard deviation \\
\hline cOrg_gcm3 & REAL & g cm-3 & Soil organic carbon content \\
\hline cOrg_percent & REAL & percent & Soil organic carbon content \\
\hline cSigma_kgm2 & REAL & $\mathrm{kg} \mathrm{m}-2$ & Soil carbon content error estimate as standard deviation \\
\hline c_kgm2 & REAL & $\mathrm{kg} \mathrm{m}-2$ & Soil carbon content \\
\hline c_percent & REAL & percent & Soil carbon content \\
\hline cec_reqg & REAL & Heq g-1 & Soil cation exchange capacity \\
\hline claySigma_percent & REAL & percent & Soil clay particle content error estimate as standard deviation \\
\hline clay_percent & REAL & percent & Soil clay particle content \\
\hline $\mathrm{cn}$ & REAL & adimensional & Soil C:N ratio \\
\hline densitySigma_gcm3 & REAL & g cm-3 & Soil bulk density content error estimate as standard deviation \\
\hline
\end{tabular}




\begin{tabular}{|c|c|c|c|}
\hline density_gcm3 & REAL & g cm-3 & Soil bulk density \\
\hline fcapv_percent & REAL & percent & Soil field capacity \\
\hline fineRoot_percent & REAL & percent & Distribution of fine roots accross soil horizons \\
\hline gravel_percent & REAL & percent & Soil gravel particle content \\
\hline horizon & TEXT & adimensional & Name of soil horizon \\
\hline humus_tCha & REAL & tC ha-1 & Humus carbon content \\
\hline hydCondSat_cmd1 & REAL & $\mathrm{cm} \mathrm{d}-1$ & Soil hydraulic conductivity at saturation \\
\hline layer_id & $\begin{array}{l}\text { INTEGE } \\
\mathrm{R}\end{array}$ & adimensional & Layer code as decimal number (1-99) \\
\hline lowerDepth_cm & REAL & $\mathrm{cm}$ & Lower soil horizon limit \\
\hline mbCSigma_mgg & REAL & $\begin{array}{l}\text { mg C g-1 dry } \\
\text { soil }\end{array}$ & Soil microbial biomass carbon error estimate as standard deviation \\
\hline mbC_mgg & REAL & $\begin{array}{l}\text { mg C g-1 dry } \\
\text { soil }\end{array}$ & Soil microbial biomass carbon \\
\hline mbNSigma_mgNg & REAL & $\begin{array}{l}\text { mg N g-1 dry } \\
\text { soil }\end{array}$ & Soil microbial biomass nitrogen error estimate as standard deviation \\
\hline mbN_mgNg & REAL & $\begin{array}{l}\text { mg N g-1 dry } \\
\text { soil }\end{array}$ & Soil microbial biomass nitrogen \\
\hline minRSigma_mgkgh & REAL & mg N kg-1 h-1 & Soil mineralisation rate error estimate as standard deviation \\
\hline minR_mgkgh & REAL & mg N kg-1 h-1 & Soil mineralisation rate \\
\hline nMax_percent & REAL & percent & Maximum soil nitrogen content \\
\hline nMin_percent & REAL & percent & Minimum soil nitrogen content \\
\hline nOrgSigma_percent & REAL & percent & Soil organic nitrogen content error estimate as standard deviation \\
\hline nOrg_percent & REAL & percent & Soil organic nitrogen content \\
\hline
\end{tabular}




\begin{tabular}{|c|c|c|c|}
\hline n_kgm2 & REAL & $\mathrm{kg} \mathrm{m}-2$ & Soil nitrogen content \\
\hline n_percent & REAL & percent & Soil nitrogen content \\
\hline ofhC_percent & REAL & percent & $\begin{array}{l}\text { The organic fermentative-humic (Ofh) subhorizon consists of forest litter (leaves, bark, twigs etc) showing } \\
\text { considerable decay. }\end{array}$ \\
\hline ofhN_percent & REAL & percent & Carbon content in a gram of OFH sample \\
\hline ofh_gDWm2 & REAL & g DW m-2 & Litter layer (leaves not decomposed) \\
\hline ol_gDWm2 & REAL & g DW m-2 & Nitrogen content in a gram of OFH sample \\
\hline phSigma_h2o & REAL & adimensional & Soil $\mathrm{pH}$ determined with $\mathrm{H} 2 \mathrm{O}$ error estimate as standard deviation \\
\hline phSigma_kcl & REAL & adimensional & Soil $\mathrm{pH}$ determined by $\mathrm{KCl}$ error estimate as standard deviation \\
\hline ph_cacl2 & REAL & adimensional & Soil $\mathrm{pH}$ determined with $\mathrm{CaCl} 2$ \\
\hline ph_h2o & REAL & adimensional & Soil $\mathrm{pH}$ determined with $\mathrm{H} 2 \mathrm{O}$ \\
\hline ph_kcl & REAL & adimensional & Soil pH deterimed with $\mathrm{KCl}$ \\
\hline porosity_percent & REAL & percent & Soil water content at saturation in the bulk soil \\
\hline rainGroundWater & REAL & adimensional & Whether the soil is mostly influenced by rain or ground water \\
\hline sandSigma_percent & REAL & percent & Soil sand particle content error estimate as standard deviation \\
\hline sand_percent & REAL & percent & Soil sand particle content \\
\hline siltSigma_percent & REAL & percent & Soil silt particle content error estimate as standard deviation \\
\hline silt_percent & REAL & percent & Soil silt particle content \\
\hline table_id & $\begin{array}{l}\text { INTEGE } \\
\text { R }\end{array}$ & adimensional & Table code as decimal number (1-99) \\
\hline texture & TEXT & adimensional & Soil texture \\
\hline thicknesSigma_cm & REAL & $\mathrm{cm}$ & Soil thickness error estimate \\
\hline thickness_cm & REAL & $\mathrm{cm}$ & Soil thickness \\
\hline
\end{tabular}




\begin{tabular}{|l|l|l|l|}
\hline type_fao & TEXT & adimensional & $\begin{array}{l}\text { Soil type after ISSS-ISRIC-FAO (1998) World reference basis for soil resources. World Soil Resources } \\
\text { Reports 84. FAO, Rome. 92 p. }\end{array}$ \\
\hline type_ka5 & TEXT & adimensional & $\begin{array}{l}\text { Soil type after AG Boden (2005) Bodenkundliche Kartieranleitung. Bundesanstalt für Geowissenschaften } \\
\text { und Rohstoffe, Hannover }\end{array}$ \\
\hline upperDepth_cm & REAL & $\mathrm{cm}$ & Upper soil horizon limit \\
\hline whcSigma_mm & REAL & $\mathrm{mm}$ & Soil water holding capacity error estimate \\
\hline whc_mm & REAL & $\mathrm{mm}$ & Soil water holding capacity \\
\hline whcp_percent & REAL & percent & Water holding capacity for plant available water \\
\hline wiltp_percent & REAL & percent & Soil wilting point \\
\hline
\end{tabular}


Table SOM3: Description of CLIMATE variables included in the database.

\begin{tabular}{|c|c|c|c|}
\hline variable & type & units & description \\
\hline record_id & INTEGER & adimensional & Record ID as decimal number \\
\hline site & TEXT & adimensional & Site name \\
\hline site_id & INTEGER & adimensional & Site code as decimal number (01-99) \\
\hline date & TEXT & adimensional & Date in format YYYY-MM-DD \\
\hline year & INTEGER & YYYY & Year with century as decimal number (0000-9999) \\
\hline mo & INTEGER & MM & Month as decimal number (01-12) \\
\hline day & INTEGER & DD & Day of the month as decimal number (01-31) \\
\hline airpress_hPa & REAL & $\mathrm{hPa}$ & Mean daily air pressure \\
\hline p_mm & REAL & $\mathrm{mm}$ & Total daily precipitation \\
\hline rad_Jcm2day & REAL & $\mathrm{J} \mathrm{cm}-2$ & Total daily global radiation \\
\hline relhum_percent & REAL & percent & Mean daily relative humidity \\
\hline tmax_degC & REAL & degree Celsius & Maximum daily temperature \\
\hline tmean_degC & REAL & degree Celsius & Mean daily temperature \\
\hline tmin_degC & REAL & degree Celsius & Minimum daily temperature \\
\hline wind_ms & REAL & m s-1 & Mean daily wind speed \\
\hline
\end{tabular}


Table SOM4: Description of CLIMATE_ISIMIP additional variables included in the database.

\begin{tabular}{|l|l|l|l|}
\hline variable & type & units & description \\
\hline site & TEXT & adimensional & Site name \\
\hline $\begin{array}{l}\text { forcingCondition } \\
\text { s }\end{array}$ & TEXT & adimensional & $\begin{array}{l}\text { This category refers to the conditions underlying the climatic forcing, e.g. following historical CO2 time series, } \\
\text { preindustrial picontrol runs or representative concentration pathways (rcp). }\end{array}$ \\
\hline forcingDataset & TEXT & adimensional & $\begin{array}{l}\text { This category refers to data taken from bias-corrected general circulation models (e.g. hadgem) or historical global } \\
\text { meteorological forcing data based on bias-corrected reanalysis data (e.g. watch) }\end{array}$ \\
\hline
\end{tabular}


Table SOM5: Description of CO2_ISIMIP variables included in the database.

\begin{tabular}{|l|l|l|l|}
\hline variable & type & units & description \\
\hline record_id & $\begin{array}{l}\text { INTEGE } \\
\text { R }\end{array}$ & adimensional & Record ID as decimal number \\
\hline site & TEXT & adimensional & Site name \\
\hline site_id & $\begin{array}{l}\text { INTEGE } \\
\text { R }\end{array}$ & adimensional & Site code as decimal number (01-99) \\
\hline $\begin{array}{l}\text { forcingCondition } \\
\text { s }\end{array}$ & TEXT & adimensional & $\begin{array}{l}\text { This category refers to the conditions underlying the climatic forcing, e.g. following historical CO2 time series or } \\
\text { representative concentration pathways (rcp). }\end{array}$ \\
\hline year & $\begin{array}{l}\text { INTEGE } \\
\text { R }\end{array}$ & YYYY & Year with century as decimal number (0000-9999) \\
\hline co2_ppm & REAL & ppm & $\begin{array}{l}\text { CO2 mean global concentrations for the different different forcing conditions: RCP and historical values (1975- } \\
\text { 2013) }\end{array}$ \\
\hline
\end{tabular}


Table SOM6: Description of NDEPOSITION and NDEPOSITION_ISIMIP2B variables included in the database.

\begin{tabular}{|l|l|l|l|}
\hline variable & type & units & description \\
\hline record_id & INTEGER & adimensional & Record ID as decimal number \\
\hline site & TEXT & adimensional & Name of the site \\
\hline site_id & INTEGER & adimensional & Site code as decimal number (01-99) \\
\hline year & INTEGER & YYYY & Year with century as decimal number (0000-9999) \\
\hline nhx_gm2 & REAL & g m-2 & Total deposition of reduced nitrogen (Dry+Wet RdN) \\
\hline noy_gm2 & REAL & g m-2 & Total deposition of oxidized nitrogen (Dry+Wet oxN) \\
\hline $\begin{array}{l}\text { forcingCondition } \\
\text { s }\end{array}$ & TEXT & adimensional & $\begin{array}{l}\text { This category refers to the conditions underlying the climatic forcing, e.g. following historical CO2 time series, } \\
\text { preindustrial picontrol runs or representative concentration pathways (rcp). }\end{array}$ \\
\hline
\end{tabular}


Table SOM7: Description of TREE variables included in the database.

\begin{tabular}{|l|l|l|l|}
\hline variable & type & units & description \\
\hline record_id & INTEGER & adimensional & Record ID as decimal number \\
\hline site & TEXT & adimensional & Site name \\
\hline site_id & INTEGER & adimensional & Site code as decimal number (01-99) \\
\hline species & TEXT & adimensional & Species name \\
\hline species_id & TEXT & adimensional & Species text code \\
\hline year & INTEGER & YYYY & Year with century as decimal number (0000-9999) \\
\hline dbh1_cm & REAL & $\mathrm{cm}$ & Diameter at breast height \\
\hline height1_m & REAL & $\mathrm{m}$ & Tree height \\
\hline size_m2 & REAL & $\mathrm{m} 2$ & Plot size \\
\hline
\end{tabular}


Table SOM8: Description of STAND variables included in the database.

\begin{tabular}{|c|c|c|c|}
\hline variable & type & units & description \\
\hline record_id & $\begin{array}{l}\text { INTEGE } \\
\text { R }\end{array}$ & adimensional & Record ID as decimal number \\
\hline site & TEXT & adimensional & Site name \\
\hline site_id & $\begin{array}{l}\text { INTEGE } \\
\text { R }\end{array}$ & adimensional & Site code as decimal number (01-99) \\
\hline species & TEXT & adimensional & Species name \\
\hline species_id & TEXT & adimensional & Species text code \\
\hline year & $\begin{array}{l}\text { INTEGE } \\
\text { R }\end{array}$ & YYYY & Year with century as decimal number (0000-9999) \\
\hline aboveGroundBiomass_kgha & REAL & kg ha-1 & Above ground biomass \\
\hline age & $\begin{array}{l}\text { INTEGE } \\
\text { R }\end{array}$ & years & Mean stand age \\
\hline ba_m2ha & REAL & m2 ha-1 & Basal area per hectare \\
\hline branchesBiomass_kgha & REAL & kg ha-1 & Branches biomass \\
\hline dbhArith_cm & REAL & $\mathrm{cm}$ & Arithmetic mean diameter \\
\hline dbhBA_cm & REAL & $\mathrm{cm}$ & $\begin{array}{l}\text { Average diameter weighted by basal area calculated as dbhBA }=(\text { ba1*dbh1 }+ \text { ba2*dbh } 2+\ldots+ \\
\text { bak*dbhk) } /(\text { ba1 }+ \text { ba } 2+\ldots+\text { bak }) \text {, where bai and dbhi are the basal area and dbh, respectively, of } \\
\text { the tree } i \text {, and } i=1,2, \ldots, k\end{array}$ \\
\hline dbhDQ_cm & REAL & $\mathrm{cm}$ & $\begin{array}{l}\text { Mean squared diameter or quadratic mean diameter calculated as } \mathrm{dbhDQ}=\operatorname{sqrt}\left(\left(\mathrm{dbh} 1^{\wedge} 2+\right.\right. \\
\left.\left.\mathrm{dbh} 2^{\wedge} 2+\ldots+\mathrm{dbhk^{ \wedge }} 2\right) / \mathrm{N}\right), \text { where } \mathrm{dbhi} \text { is the diameter at breat height of tree } \mathrm{i}, \mathrm{i}=1,2, \ldots, \mathrm{k}, \mathrm{N} \text { is } \\
\text { the total number of trees, and sqrt is the square root }\end{array}$ \\
\hline density_treeha & REAL & tree ha-1 & Number of tree per ha \\
\hline foliageBiomass_kgha & REAL & kg ha-1 & Foliage biomass \\
\hline
\end{tabular}




\begin{tabular}{|l|l|l|l|}
\hline heightArith_m & REAL & $\mathrm{m}$ & Arithmetic mean height \\
\hline heightBA_m & REAL & $\mathrm{m}$ & $\begin{array}{l}\text { Average height weighted by basal area or Loreys height calculated as heightBA }=(\mathrm{ba1} \text { *h1 }+ \\
\text { ba2*h2 }+\ldots+\text { bak*hk }) /(\mathrm{ba} 1+\mathrm{ba2}+\ldots+\text { bak }), \text { where bai and hi are the basal area and height, } \\
\text { respectively, of the tree } \mathrm{i}, \text { and } \mathrm{i}=1,2, \ldots, \mathrm{k}\end{array}$ \\
\hline lai & REAL & adimensional & Leaf Area Index \\
\hline rootBiomass_kgha & REAL & $\mathrm{kg}$ ha-1 & Root biomass \\
\hline stemBiomass_kgha & REAL & $\mathrm{kg}$ ha-1 & Stem biomass \\
\hline $\begin{array}{l}\text { stumpCoarseRootBiomass_kgh } \\
\text { a }\end{array}$ & REAL & kg ha-1 & Stump and coarse roots biomass \\
\hline
\end{tabular}


Table SOM9: Description of FLUX variables included in the database.

\begin{tabular}{|c|c|c|c|}
\hline variable & type & units & description \\
\hline record_id & INTEGER & adimensional & Record ID as decimal number \\
\hline site_id & INTEGER & adimensional & Site code as decimal number (01-99) \\
\hline date & TEXT & adimensional & Date in format YYYY-MM-DD hh:mm:ss. Derived from TIMESTAMP_START \\
\hline year & INTEGER & YYYY & Year with century as decimal number (0000-9999). Derived from TIMESTAMP_START \\
\hline mo & INTEGER & MM & Month as decimal number (01-12). Derived from TIMESTAMP_START \\
\hline day & INTEGER & DD & Day of the month as decimal number (01-31). Derived from TIMESTAMP_START \\
\hline gppDtCutRef_umolCO2m2s1 & REAL & umolCO2 m-2 s-1 & $\begin{array}{l}\text { Gross Primary Production, from Daytime partitioning method, reference selected from } \\
\text { GPP versions using a model efficiency approach. Based on corresponding } \\
\text { NEE_CUT_XX version }\end{array}$ \\
\hline gppDtCutSe_umolCO2m2s1 & REAL & umolCO2 m-2 s-1 & $\begin{array}{l}\text { Standard Error for Gross Primary Production, calculated as stdev(gppDtCut_XX) / } \\
\text { sqrt(40). SE from } 40 \text { half-hourly gppDtCut_XX }\end{array}$ \\
\hline gppDtVutRef_umolCO2m2s1 & REAL & umolCO2 m-2 s-1 & $\begin{array}{l}\text { Gross Primary Production, from Daytime partitioning method, reference version selected } \\
\text { from GPP versions using a model efficiency approach. Based on corresponding } \\
\text { neeVut_XX version }\end{array}$ \\
\hline gppDtVutSe_umolCO2m2s1 & REAL & umolCO2 m-2 s-1 & $\begin{array}{l}\text { Standard Error for Gross Primary Production, calculated as stdev(gppDtVut_XX) / } \\
\text { sqrt(40. SE from } 40 \text { half-hourly gppDtVut_XX }\end{array}$ \\
\hline gppNtCutRef_umolCO2m2s1 & REAL & umolCO2 m-2 s-1 & $\begin{array}{l}\text { Gross Primary Production, from Nighttime partitioning method, reference selected from } \\
\text { GPP versions using a model efficiency approach. Based on corresponding } \\
\text { NEE_CUT_XX version }\end{array}$ \\
\hline gppNtCutSe_umolCO2m2s1 & REAL & umolCO2 m-2 s-1 & $\begin{array}{l}\text { Standard Error for Gross Primary Production, calculated as stdev(gppNtCut_XX) / } \\
\text { sqrt(40). SE from } 40 \text { half-hourly gppNtCut_XX }\end{array}$ \\
\hline gppNtVutRef_umolCO2m2s1 & REAL & umolCO2 m-2 s-1 & $\begin{array}{l}\text { Gross Primary Production, from Nighttime partitioning method, reference version } \\
\text { selected from GPP versions using a model efficiency approach. Based on corresponding } \\
\text { neeVut_XX version }\end{array}$ \\
\hline
\end{tabular}




\begin{tabular}{|c|c|c|c|}
\hline gppNtVutSe_umolCO2m2s1 & REAL & umolCO2 m-2 s-1 & $\begin{array}{l}\text { Standard Error for Gross Primary Production, calculated as (stdev(gppNtVut_XX) / } \\
\text { sqrt(40)). SE from } 40 \text { half-hourly gppNtVut_XX }\end{array}$ \\
\hline $\begin{array}{l}\text { neeCutRefJointunc_umolCO2m2s } \\
1\end{array}$ & REAL & umolCO2 m-2 s-1 & $\begin{array}{l}\text { Joint uncertainty estimation for neeCutRef, including random uncertainty and USTAR } \\
\text { filtering uncertainty [sqrt(neeCutRef_RANDUNC^2 + ((NEE_CUT_84 - NEE_CUT_16) / } \\
\text { 2)^2)] for each half-hour }\end{array}$ \\
\hline neeCutRef_qc & INTEGER & adimensional & Quality flag for neeCutRef. $0=$ measured; $1=$ good quality gapfill; $2=$ medium; $3=$ poor. \\
\hline neeCutRef_umolCO2m2s1 & REAL & umolCO2 m-2 s-1 & $\begin{array}{l}\text { Net Ecosystem Exchange, using Constant Ustar Threshold (CUT) across years, } \\
\text { reference selected on the basis of the model efficiency }\end{array}$ \\
\hline $\begin{array}{l}\text { neeVutRefJointunc_umolCO2m2s } \\
1\end{array}$ & REAL & umolCO2 m-2 s-1 & $\begin{array}{l}\text { Joint uncertainty estimation for neeVutRef, including random uncertainty and USTAR } \\
\text { filtering uncertainty [sqrt(neeVutRef_RANDUNC^2 + ((neeVut_84 - neeVut_16) / 2)^2)] } \\
\text { for each half-hour }\end{array}$ \\
\hline neeVutRef_qc & INTEGER & adimensional & Quality flag for neeVutRef. $0=$ measured; $1=$ good quality gapfill; $2=$ medium; $3=$ poor. \\
\hline neeVutRef_umolCO2m2s1 & REAL & umolCO2 m-2 s-1 & $\begin{array}{l}\text { Net Ecosystem Exchange, using Variable Ustar Threshold (VUT) for each year, } \\
\text { reference selected on the basis of the model efficiency }\end{array}$ \\
\hline recoDtCutRef_umolCO2m2s1 & REAL & umolCO2 m-2 s-1 & $\begin{array}{l}\text { Ecosystem Respiration, from Daytime partitioning method, reference selected from } \\
\text { RECO versions using a model efficiency approach. Based on corresponding } \\
\text { NEE_CUT_XX version }\end{array}$ \\
\hline recoDtCutSe_umolCO2m2s1 & REAL & umolCO2 m-2 s-1 & $\begin{array}{l}\text { Standard Error for Ecosystem Respiration, calculated as stdev(recoDtCut_XX) / sqrt(40). } \\
\text { SE from } 40 \text { half-hourly recoDtCut_XX }\end{array}$ \\
\hline recoDtVutRef_umolCO2m2s1 & REAL & umolCO2 m-2 s-1 & $\begin{array}{l}\text { Ecosystem Respiration, from Daytime partitioning method, reference selected from } \\
\text { RECO versions using a model efficiency approach. Based on corresponding neeVut_XX } \\
\text { version }\end{array}$ \\
\hline recoDtVutSe_umolCO2m2s1 & REAL & umolCO2 m-2 s-1 & $\begin{array}{l}\text { Standard Error for Ecosystem Respiration, calculated as stdev(recoDtVut_XX) / sqrt(40). } \\
\text { SE from } 40 \text { half-hourly recoDtCut_XX }\end{array}$ \\
\hline recoNtCutRef_umolCO2m2s1 & REAL & umolCO2 m-2 s-1 & $\begin{array}{l}\text { Ecosystem Respiration, from Nighttime partitioning method, reference selected from } \\
\text { RECO versions using a model efficiency approach. Based on corresponding } \\
\text { NEE_CUT_XX version }\end{array}$ \\
\hline recoNtCutSe_umolCO2m2s1 & REAL & umolCO2 m-2 s-1 & $\begin{array}{l}\text { Standard Error for Ecosystem Respiration, calculated as stdev(recoNtCut_XX) / sqrt(40. } \\
\text { SE from } 40 \text { half-hourly recoNtCut XX }\end{array}$ \\
\hline
\end{tabular}




\begin{tabular}{|l|l|l|l|}
\hline recoNtVutRef_umolCO2m2s1 & REAL & umolCO2 m-2 s-1 & $\begin{array}{l}\text { Ecosystem Respiration, from Nighttime partitioning method, reference selected from } \\
\text { RECO versions using a model efficiency approach. Based on corresponding neeVut_XX } \\
\text { version }\end{array}$ \\
\hline recoNtVutSe_umolCO2m2s1 & REAL & umolCO2 m-2 s-1 & $\begin{array}{l}\text { Standard Error for Ecosystem Respiration, calculated as stdev(recoNtVut_XX)/ sqrt(40. } \\
\text { SE from 40 half-hourly recoNtCut_XX }\end{array}$ \\
\hline timestampEnd & TEXT & YYYYMMDDHHMM & ISO timestamp end of averaging period - short format \\
\hline timestampStart & TEXT & YYYYMMDDHHMM & ISO timestamp start of averaging period - short format \\
\hline
\end{tabular}


Table SOM10: Description of MODIS variables included in the database.

\begin{tabular}{|c|c|c|c|c|c|}
\hline variable & type & units & description & comments & source \\
\hline record_id & INTEGER & adimensional & Record ID as decimal number & & \\
\hline site & TEXT & adimensional & Site name & & \\
\hline site_id & INTEGER & adimensional & Site code as decimal number (01-99) & & \\
\hline date & TEXT & adimensional & Date in format YYYY-MM-DD & & \\
\hline year & INTEGER & YYYY & $\begin{array}{l}\text { Year with century as decimal number (0000- } \\
\text { 9999) }\end{array}$ & & \\
\hline mo & INTEGER & MM & Month as decimal number (01-12) & & \\
\hline day & INTEGER & DD & Day of the month as decimal number (01-31) & & \\
\hline aB01_rad & REAL & radian & $\begin{array}{l}\text { Angle in red. Spatial resolution: } 0.5 \mathrm{~km} \text {. } \\
\text { Temporal resolution: 8-day composite. }\end{array}$ & $\begin{array}{l}\text { Calculated from MOD09A1 after Khanna, S., } \\
\text { A. Palacios-Orueta, M. L. Whiting, S. L. Ustin, } \\
\text { D. R. no, and J. Litago, 2007. Development of } \\
\text { angle indexes for soil moisture estimation, dry } \\
\text { matter detection and land-cover } \\
\text { discrimination. Remote Sensing of } \\
\text { Environment 109:154 - 165. Note: The } \\
\text { observations with values larger than (average } \\
+ \text { 1standard deviation) or smaller than } \\
\text { (average - 1standard deviation) in a window } \\
\text { size of } 5 \text { observations have been filtered and } \\
\text { substituted with the average value of the } \\
\text { window. }\end{array}$ & MOD09A1 \\
\hline aB02_rad & REAL & radian & $\begin{array}{l}\text { Angle in near infrared. Calculated with } \\
\text { MOD09A1. Spatial resolution } 0.5 \mathrm{~km}\end{array}$ & $\begin{array}{l}\text { Calculated from MOD09A1 after Palacios- } \\
\text { Orueta, A., M. Huesca, M. L. Whiting, J. } \\
\text { Litago, S. Khanna, M. Garcia, and S. L. Ustin, } \\
\text { 2012. Derivation of phenological metrics by } \\
\text { function fitting to time-series of spectral shape } \\
\text { indexes AS1 and AS2: Mapping cotton } \\
\text { phenological stages using modis time series. }\end{array}$ & MOD09A1 \\
\hline
\end{tabular}




\begin{tabular}{|c|c|c|c|c|c|}
\hline & & & & $\begin{array}{l}\text { Remote Sensing of Environment 12:148-159. } \\
\text { Note: The observations with values larger } \\
\text { than (average }+1 \text { standard deviation) or } \\
\text { smaller than (average }-1 \text { standard deviation) } \\
\text { in a window size of } 5 \text { observations have been } \\
\text { filtered and substituted with the average value } \\
\text { of the window. }\end{array}$ & \\
\hline aB05_rad & REAL & radian & $\begin{array}{l}\text { Angle in SWIR 1.Spatial resolution: } 0.5 \mathrm{~km} \text {. } \\
\text { Temporal resolution: 8-day composite. }\end{array}$ & $\begin{array}{l}\text { Calculated from MOD09A1 after Palacios- } \\
\text { Orueta, A., M. Huesca, M. L. Whiting, J. } \\
\text { Litago, S. Khanna, M. Garcia, and S. L. Ustin, } \\
\text { 2012. Derivation of phenological metrics by } \\
\text { function fitting to time-series of spectral shape } \\
\text { indexes AS1 and AS2: Mapping cotton } \\
\text { phenological stages using modis time series. } \\
\text { Remote Sensing of Environment 12:148-159. } \\
\text { Note: The observations with values larger } \\
\text { than (average + 1standard deviation) or } \\
\text { smaller than (average - 1standard deviation) } \\
\text { in a window size of } 5 \text { observations have been } \\
\text { filtered and substituted with the average value } \\
\text { of the window. }\end{array}$ & MOD09A1 \\
\hline aB06_rad & REAL & radian & $\begin{array}{l}\text { Angle in SWIR 2. Spatial resolution: } 0.5 \mathrm{~km} \text {. } \\
\text { Temporal resolution: 8-day composite. }\end{array}$ & $\begin{array}{l}\text { Calculated from MOD09A1 after Palacios- } \\
\text { Orueta, A., M. Huesca, M. L. Whiting, J. } \\
\text { Litago, S. Khanna, M. Garcia, and S. L. Ustin, } \\
\text { 2012. Derivation of phenological metrics by } \\
\text { function fitting to time-series of spectral shape } \\
\text { indexes AS1 and AS2: Mapping cotton } \\
\text { phenological stages using modis time series. } \\
\text { Remote Sensing of Environment 12:148-159. } \\
\text { Note: The observations with values larger } \\
\text { than (average + 1standard deviation) or } \\
\text { smaller than (average - 1standard deviation) }\end{array}$ & MOD09A1 \\
\hline
\end{tabular}




\begin{tabular}{|c|c|c|c|c|c|}
\hline & & & & $\begin{array}{l}\text { in a window size of } 5 \text { observations have been } \\
\text { filtered and substituted with the average value } \\
\text { of the window. }\end{array}$ & \\
\hline evi16 & REAL & adimensional & $\begin{array}{l}\text { Enhanced Vegetation Index. Valid range: }-0.2 \text { - } \\
\text { 1. Fill value: NA. Spatial resolution: } 250 \text { meters. } \\
\text { Temporal resolution: } 16 \text {-day composite. }\end{array}$ & $\begin{array}{l}\text { Data are provided every } 16 \text { days at } 250 \text {-meter } \\
\text { spatial resolution. Each value corresponds to } \\
\text { the best observation during a } 16 \text { day period. }\end{array}$ & MOD13Q1 \\
\hline evi16_qc & REAL & adimensional & $\begin{array}{l}\text { Indicates the level of the product quality that is } \\
\text { classified as follows: } 0=\text { good quality, index } \\
\text { produced; } 2=\text { other quality, index produced, but } \\
\text { check other qc and index produced, but most } \\
\text { probably cloudy; } 3=\text { index not produced due to } \\
\text { other reasons than cloud, thus fill values were } \\
\text { substituted by an interpolated values when the } \\
\text { previous and the following values were available } \\
\text { index = (indext- } 1+\text { indext }+1) / 2 \text {. }\end{array}$ & & MOD13Q1 \\
\hline evi8 & REAL & adimensional & $\begin{array}{l}\text { Enhance Vegetation Index. Spatial resolution: } \\
0.5 \mathrm{~km} \text {. Temporal resolution: 8-day composite }\end{array}$ & & MOD09A1 \\
\hline fpar & REAL & adimensional & $\begin{array}{l}\text { Proportion of available radiation in the } \\
\text { photosynthetically active wavelengths. Valid } \\
\text { range: } 0 \text { - } 1 \text {. Fill value: NA. Spatial resolution: } 1 \\
\text { km. Temporal resolution: 8-day composite. }\end{array}$ & & MOD15A2 \\
\hline fpar_qc & INTEGER & adimensional & $\begin{array}{l}\text { Indicates the level of the product quality that is } \\
\text { classified as follows: } 0=\text { Good quality (main } \\
\text { algorithm with or without saturation); } 2=\text { Other } \\
\text { quality (back-up algorithm or fill values) }\end{array}$ & & MOD15A2 \\
\hline gpp_gCm2d & REAL & gC m-2 d-1 & $\begin{array}{l}\text { Gross Primary Production. Valid range: }-375 \text { - } \\
\text { 375. Fill value: NA. Spatial resolution } 1 \mathrm{~km} \text {. } \\
\text { Temporal resolution: 8-day accumulation. }\end{array}$ & $\begin{array}{l}\text { This has been calculated by dividing each } 8 \text { - } \\
\text { day values by } 8 \text { for the first } 45 \text { values/year } \\
\text { and by } 5 \text { or } 6 \text { for the final period. }\end{array}$ & MOD17A2 \\
\hline gpp_qc & INTEGER & adimensional & $\begin{array}{l}\text { Indicates the level of the product quality that is } \\
\text { classified as follows: } 0=\text { good quality, the } \\
\text { estimates were done using the main algorithm }\end{array}$ & & MOD17A2 \\
\hline
\end{tabular}




\begin{tabular}{|c|c|c|c|c|c|}
\hline & & & $\begin{array}{l}\text { with or without saturation; } 2 \text { = other quality, the } \\
\text { estimates were done using back-up algorithm. }\end{array}$ & & \\
\hline lai & REAL & adimensional & $\begin{array}{l}\text { Leaf area index. Valid range: } 0 \text { - 10. Fill value: } \\
\text { NA. Spatial resolution: } 1 \mathrm{~km} \text {. Temporal } \\
\text { resolution: 8-day composite. }\end{array}$ & & MOD15A2 \\
\hline lai_qc & INTEGER & adimensional & $\begin{array}{l}\text { Indicates the level of the product quality that is } \\
\text { classified as follows: } 0=\text { Good quality (main } \\
\text { algorithm with or without saturation); } 2=\text { Other } \\
\text { quality (back-up algorithm or fill values) }\end{array}$ & & MOD15A2 \\
\hline IstDay_degK & REAL & degree Kelvin & $\begin{array}{l}\text { Daytime land surface temperature. Valid range: } \\
150 \text { - 1310.7. Fill value: NA. Spatial resolution: } 1 \\
\text { km. Temporal resolution: 8-day composite. }\end{array}$ & $\begin{array}{l}\text { The level-3 MODIS global Land Surface } \\
\text { Temperature (LST) and Emissivity 8-day data } \\
\text { are composed from the daily 1-kilometer LST } \\
\text { product (MOD11A1) the average values of } \\
\text { clear-sky LSTs during an 8-day period. In this } \\
\text { data set the daytime and nighttime LSTs, are } \\
\text { provided. Products are validated to Stage } 2 \text {, } \\
\text { which means that their accuracy has been } \\
\text { assessed over a widely distributed set of } \\
\text { locations and time periods via several ground- } \\
\text { truth and validation efforts. }\end{array}$ & MOD11A2 \\
\hline IstDay_qc & INTEGER & adimensional & $\begin{array}{l}\text { Indicates the level of quality of the product that is } \\
\text { classified as follows: } 0=\text { good quality; } 2=\text { other } \\
\text { quality; } 3=\text { interpolated, } 4=\text { pixel not produced } \\
\text { (NA) }\end{array}$ & & MOD11A2 \\
\hline IstNight_degK & REAL & degree Kelvin & $\begin{array}{l}\text { Nighttime land surface temperature. Valid range: } \\
150 \text { - 1310.7. Fill value: NA. Spatial resolution: } 1 \\
\mathrm{~km} \text {. Temporal resolution: 8-day composite. }\end{array}$ & $\begin{array}{l}\text { The level-3 MODIS global Land Surface } \\
\text { Temperature (LST) and Emissivity 8-day data } \\
\text { are composed from the daily 1-kilometer LST } \\
\text { product (MOD11A1) the average values of } \\
\text { clear-sky LSTs during an 8-day period. In this } \\
\text { data set the daytime and nighttime LSTs, are } \\
\text { provided. Products are validated to Stage 2, }\end{array}$ & MOD11A2 \\
\hline
\end{tabular}




\begin{tabular}{|c|c|c|c|c|c|}
\hline & & & & $\begin{array}{l}\text { which means that their accuracy has been } \\
\text { assessed over a widely distributed set of } \\
\text { locations and time periods via several ground- } \\
\text { truth and validation efforts. }\end{array}$ & \\
\hline IstNight_qc & INTEGER & adimensional & $\begin{array}{l}\text { Indicates the level of quality of the product that is } \\
\text { classified as follows: } 0=\text { good quality; } 2=\text { other } \\
\text { quality; } 3=\text { interpolated, } 4=\text { pixel not produced } \\
\text { (NA) }\end{array}$ & & MOD11A2 \\
\hline ndvi16 & REAL & adimensional & $\begin{array}{l}\text { Normalized Difference Vegetation Index. Valid } \\
\text { range: }-0.2 \text { - } 1 \text {. Fill value: NA. Spatial resolution: } \\
250 \text { meters. Temporal resolution: } 16 \text {-day } \\
\text { composite. }\end{array}$ & $\begin{array}{l}\text { Data are provided every } 16 \text { days at } 250 \text {-meter } \\
\text { spatial resolution. Each value corresponds to } \\
\text { the best observation during a } 16 \text { day period. }\end{array}$ & MOD13Q1 \\
\hline ndvi16_qc & REAL & adimensional & $\begin{array}{l}\text { Indicates the level of the product quality that is } \\
\text { classified as follows: } 0=\text { good quality, index } \\
\text { produced; } 2=\text { other quality, index produced, but } \\
\text { check other qc and index produced, but most } \\
\text { probably cloudy; } 3=\text { index not produced due to } \\
\text { other reasons than cloud, thus fill values were } \\
\text { substituted by an interpolated values when the } \\
\text { previous and the following values were available } \\
\text { index = (indext- } 1+\text { indext }+1) / 2 \text {. }\end{array}$ & & MOD13Q1 \\
\hline ndvi8 & REAL & adimensional & $\begin{array}{l}\text { Normalized Difference Vegetation Index. Spatial } \\
\text { resolution: } 0.5 \mathrm{~km} \text {. Temporal resolution: 8-day } \\
\text { composite }\end{array}$ & $\begin{array}{l}\text { Calculated from MOD09A1 after Tucker, C.J., } \\
\text { 1979. Red and photographic infrared linear } \\
\text { combinations for monitoring vegetation. } \\
\text { Remote Sensing of Environment. 8, 127-150. } \\
\text { Note: The observations with values larger } \\
\text { than (average + 1standard deviation) or } \\
\text { smaller than (average - 1standard deviation) } \\
\text { in a window size of } 5 \text { observations have been } \\
\text { filtered and substituted with the average value } \\
\text { of the window. }\end{array}$ & MOD09A1 \\
\hline
\end{tabular}




\begin{tabular}{|c|c|c|c|c|c|}
\hline ndwi & REAL & adimensional & $\begin{array}{l}\text { Normalized Difference Water Index. Spatial } \\
\text { resolution: } 0.5 \mathrm{~km} \text {. Temporal resolution: 8-day } \\
\text { composite }\end{array}$ & $\begin{array}{l}\text { Calculated from MOD09A1 after Gao, B., } \\
\text { 1996. NDWI-a normalized difference water } \\
\text { index for remote sensing of vegetation liquid } \\
\text { water from space. Remote Sensing of } \\
\text { Environment. 58, 257-266. Note: The } \\
\text { observations with values larger than (average } \\
+1 \text { standard deviation) or smaller than } \\
\text { (average - 1standard deviation) in a window } \\
\text { size of } 5 \text { observations have been filtered and } \\
\text { substituted with the average value of the } \\
\text { window. }\end{array}$ & MOD09A1 \\
\hline psNet_gCm2d & REAL & $g C \mathrm{~m}-2 \mathrm{~d}-1$ & $\begin{array}{l}\text { Net Photosynthesis (GPP - maintenance } \\
\text { respiration). Valid range: }-375-375 \text {. Fill value: } \\
\text { NA. Spatial resolution } 1 \mathrm{~km} \text {. Temporal } \\
\text { resolution: 8-day accumulation. }\end{array}$ & $\begin{array}{l}\text { This has been calculated by dividing each } 8 \text { - } \\
\text { day values by } 8 \text { for the first } 45 \text { values/year } \\
\text { and by } 5 \text { or } 6 \text { for the final period. }\end{array}$ & MOD17A2 \\
\hline psNet_qc & INTEGER & adimensional & $\begin{array}{l}\text { Indicates the level of the product quality that is } \\
\text { classified as follows: } 0=\text { good quality, the } \\
\text { estimates were done using the main algorithm } \\
\text { with or without saturation; } 2 \text { = other quality, the } \\
\text { estimates were done using back-up algorithm. }\end{array}$ & & MOD17A2 \\
\hline refl_qc & INTEGER & adimensional & $\begin{array}{l}\text { Indicates the level of quality correction of the } \\
\text { product (the seven bands) that is classified as } \\
\text { follows: } 0=\text { Highest quality, corrected product } \\
\text { produced at ideal quality all bands; } 2=\text { corrected } \\
\text { product produced at less than ideal quality some } \\
\text { or all bands, some bands could not be } \\
\text { completely correct; } 3=\text { interpolated, when } \\
\text { corrected product has not been produced in one } \\
\text { or some bands and they have been interpolated } \\
\text { with the value Rt = (Rt- } 1+\mathrm{Rt}+1) / 2 ; 4=\text { corrected } \\
\text { product not produced, when product has not } \\
\text { been completely corrected in one or some bands }\end{array}$ & & MOD09A1 \\
\hline
\end{tabular}




\begin{tabular}{|c|c|c|c|c|c|}
\hline & & & $\begin{array}{l}\text { and could not be interpolated. Data may be } \\
\text { wrong or filled with NA; } 5=\text { Missing data, } \\
\text { indicates that the product was not available for } \\
\text { that date. Some of them correspond to specific } \\
\text { continuous periods. All the columns filled with } \\
\text { NA. }\end{array}$ & & \\
\hline reflB01_percent & REAL & percent reflectance & $\begin{array}{l}\text { Surface Reflectance Band } 1(620-670 \mathrm{~nm}) \text { Red. } \\
\text { Fill value: NA. Spatial resolution: } 0.5 \mathrm{~km} \text {. } \\
\text { Temporal resolution: 8-day composite. }\end{array}$ & & MOD09A1 \\
\hline reflB02_percent & REAL & percent reflectance & $\begin{array}{l}\text { Surface Reflectance Band } 2(841-876 \mathrm{~nm}) \mathrm{NIR} \text {. } \\
\text { Fill value: NA. Spatial resolution: } 0.5 \mathrm{~km} . \\
\text { Temporal resolution: 8-day composite. }\end{array}$ & & MOD09A1 \\
\hline reflB03_percent & REAL & percent reflectance & $\begin{array}{l}\text { Surface Reflectance Band } 3(459-479 \mathrm{~nm}) \text { Blue. } \\
\text { Fill value: NA. Spatial resolution: } 0.5 \mathrm{~km} \text {. } \\
\text { Temporal resolution: 8-day composite. }\end{array}$ & & MOD09A1 \\
\hline reflB04_percent & REAL & percent reflectance & $\begin{array}{l}\text { Surface Reflectance Band } 4(545-565 \mathrm{~nm}) \\
\text { Green. Fill value: NA. Spatial resolution: } 0.5 \mathrm{~km} \text {. } \\
\text { Temporal resolution: 8-day composite. }\end{array}$ & & MOD09A1 \\
\hline reflB05_percent & REAL & percent reflectance & $\begin{array}{l}\text { Surface Reflectance Band } 5(1230-1250 \mathrm{~nm}) \\
\text { SWIR1. Fill value: NA. Spatial resolution: } 0.5 \mathrm{~km} \text {. } \\
\text { Temporal resolution: 8-day composite. }\end{array}$ & & MOD09A1 \\
\hline reflB06_percent & REAL & percent reflectance & $\begin{array}{l}\text { Surface Reflectance Band } 6(1628-1652 \mathrm{~nm}) \\
\text { SWIR2. Fill value: NA. Spatial resolution: } 0.5 \mathrm{~km} \text {. } \\
\text { Temporal resolution: 8-day composite. }\end{array}$ & & MOD09A1 \\
\hline reflB07_percent & REAL & percent reflectance & $\begin{array}{l}\text { Surface Reflectance Band } 7(2105-2155 \mathrm{~nm}) \\
\text { SWIR3. Fill value: NA. Spatial resolution: } 0.5 \mathrm{~km} \text {. } \\
\text { Temporal resolution: 8-day composite. }\end{array}$ & & MOD09A1 \\
\hline sani_rad & REAL & radian & $\begin{array}{l}\text { Shortwave Angle Normalized Index. Valid range: } \\
\text {-3.14 - 3.14. Spatial resolution: } 0.5 \mathrm{~km} \text {. Temporal } \\
\text { resolution: 8-day composite. }\end{array}$ & $\begin{array}{l}\text { Calculated from MOD09A1 after Palacios- } \\
\text { Orueta, A., Khanna, S., Litago, J., Whiting, } \\
\text { M.L., Ustin, S.L., 2006. Assessment of NDVI }\end{array}$ & MOD09A1 \\
\hline
\end{tabular}




\begin{tabular}{|c|c|c|c|c|c|}
\hline & & & & $\begin{array}{l}\text { and NDWI spectral indices using MODIS time } \\
\text { series analysis and development of a new } \\
\text { spectral index based on MODIS shortwave } \\
\text { infrared bands. 1st International Conference } \\
\text { on Remote Sensing and Geoinformation } \\
\text { Processing in the Assessment and Monitoring } \\
\text { of Land Degradation and Desertification, Trier, } \\
\text { Germany, pp. 207-209. Note: The } \\
\text { observations with values larger than (average } \\
+1 \text { standard deviation) or smaller than } \\
\text { (average - 1standard deviation) in a window } \\
\text { size of } 5 \text { observations have been filtered and } \\
\text { substituted with the average value of the } \\
\text { window. }\end{array}$ & \\
\hline sasi_rad & REAL & radian & $\begin{array}{l}\text { Shortwave Angle Slope Index. Spatial resolution: } \\
0.5 \mathrm{~km} \text {. Temporal resolution: 8-day composite } \\
\end{array}$ & $\begin{array}{l}\text { Calculated from MOD09A1 after Khanna, S., } \\
\text { A. Palacios-Orueta, M. L. Whiting, S. L. Ustin, } \\
\text { D. R. no, and J. Litago, 2007. Development of } \\
\text { angle indexes for soil moisture estimation, dry } \\
\text { matter detection and land-cover } \\
\text { discrimination. Remote Sensing of } \\
\text { Environment 109:154 - 165. Note: The } \\
\text { observations with values larger than (average } \\
+1 \text { standard deviation) or smaller than } \\
\text { (average - 1standard deviation) in a window } \\
\text { size of } 5 \text { observations have been filtered and } \\
\text { substituted with the average value of the } \\
\text { window. }\end{array}$ & MOD09A1 \\
\hline
\end{tabular}


Table SOM11: Description of CLIMATE_LOCAL quality variables from FLUXNET included in the database.

\begin{tabular}{|c|c|c|c|}
\hline variable & type & units & description \\
\hline site & TEXT & adimensional & Site name \\
\hline airpress_qc & REAL & adimensional & $\begin{array}{l}\text { fraction between } 0-1 \text {; indicating percentage of measured and good quality gapfill half-hourly data used to create the } \\
\text { daily value }\end{array}$ \\
\hline p_qc & REAL & adimensional & $\begin{array}{l}\text { fraction between } 0-1 \text {; indicating percentage of measured and good quality gapfill half-hourly data used to create the } \\
\text { daily value }\end{array}$ \\
\hline rad_qc & REAL & adimensional & $\begin{array}{l}\text { fraction between } 0-1 \text {; indicating percentage of measured and good quality gapfill half-hourly data used to create the } \\
\text { daily value }\end{array}$ \\
\hline relhum_qc & REAL & adimensional & $\begin{array}{l}\text { fraction between } 0-1 \text {; indicating percentage of measured and good quality gapfill half-hourly data used to create the } \\
\text { daily value }\end{array}$ \\
\hline tmax_qc & REAL & adimensional & $\begin{array}{l}\text { fraction between } 0-1 \text {; indicating percentage of measured and good quality gapfill half-hourly data used to create the } \\
\text { daily value }\end{array}$ \\
\hline tmean_qc & REAL & adimensional & $\begin{array}{l}\text { fraction between } 0-1 \text {; indicating percentage of measured and good quality gapfill half-hourly data used to create the } \\
\text { daily value }\end{array}$ \\
\hline tmin_qc & REAL & adimensional & $\begin{array}{l}\text { fraction between } 0-1 \text {; indicating percentage of measured and good quality gapfill half-hourly data used to create the } \\
\text { daily value }\end{array}$ \\
\hline wind_qc & REAL & adimensional & $\begin{array}{l}\text { fraction between } 0-1 \text {; indicating percentage of measured and good quality gapfill half-hourly data used to create the } \\
\text { daily value }\end{array}$ \\
\hline
\end{tabular}


Table SOM12: Description of ATMOSPHERIC_HEAT_CONDUCTANCE variables included in the database.

\begin{tabular}{|c|c|c|c|}
\hline variable & type & units & description \\
\hline record_id & $\begin{array}{l}\text { INTEGE } \\
\text { R }\end{array}$ & adimensional & Record ID as decimal number \\
\hline site_id & $\begin{array}{l}\text { INTEGE } \\
\text { R }\end{array}$ & adimensional & Site code as decimal number (01-99) \\
\hline date & TEXT & adimensional & Date in format YYYY-MM-DD hh:mm:ss. Derived from TIMESTAMP_START \\
\hline year & $\begin{array}{l}\text { INTEGE } \\
\mathrm{R}\end{array}$ & YYYY & Year with century as decimal number (0000-9999). Derived from TIMESTAMP_START \\
\hline mo & $\begin{array}{l}\text { INTEGE } \\
\text { R }\end{array}$ & MM & Month as decimal number (01-12). Derived from TIMESTAMP_START \\
\hline day & $\begin{array}{l}\text { INTEGE } \\
\text { R }\end{array}$ & DD & Day of the month as decimal number (01-31). Derived from TIMESTAMP_START \\
\hline $\begin{array}{l}\text { hCORRJOINTUNC_Wm } \\
2\end{array}$ & REAL & W m-2 & Joint uncertainty estimation for $\mathrm{h}$ as sqrt(hRANDUNC² + ((hCORR75 - hCORR25) / 1.349)^2) \\
\hline hCORR_Wm2 & REAL & W m-2 & Sensible heat flux, corrected hFMDS by energy balance closure correction factor \\
\hline hFMDS_Wm2 & REAL & W m-2 & Sensible heat flux, gapfilled using MDS method \\
\hline hFMDS_qc & $\begin{array}{l}\text { INTEGE } \\
\text { R }\end{array}$ & adimensional & Quality flag for hCORR. 0 = measured; 1 = good quality gapfill; 2 = medium; 3 = poor. \\
\hline $\begin{array}{l}\text { leCORRJOINTUNC_Wm } \\
2\end{array}$ & REAL & W m-2 & Joint uncertainty estimation for le \\
\hline leCORR_Wm2 & REAL & W m-2 & Latent heat flux, corrected le_FMDS by energy balance closure correction factor \\
\hline leFMDS_Wm2 & REAL & W m-2 & Latent heat flux, gapfilled using MDS method \\
\hline leFMDS_qc & $\begin{array}{l}\text { INTEGE } \\
\text { R }\end{array}$ & adimensional & Quality flag for leCORR. 0 = measured; 1 = good quality gapfill; 2 = medium; 3 = poor. \\
\hline timestampEnd & TEXT & YYYYMMDDHHMM & ISO timestamp end of averaging period - short format \\
\hline
\end{tabular}


Table SOM13: Description of SOILTS variables included in the database.

\begin{tabular}{|c|c|c|c|}
\hline variable & type & units & description \\
\hline record_id & INTEGER & adimensional & Record ID as decimal number \\
\hline site_id & INTEGER & adimensional & Site code as decimal number (01-99) \\
\hline date & TEXT & adimensional & Date in format YYYY-MM-DD hh:mm:ss. Derived from TIMESTAMP_START \\
\hline year & INTEGER & YYYY & Year with century as decimal number (0000-9999). Derived from TIMESTAMP_START \\
\hline mo & INTEGER & MM & Month as decimal number (01-12). Derived from TIMESTAMP_START \\
\hline day & INTEGER & DD & Day of the month as decimal number (01-31). Derived from TIMESTAMP_START \\
\hline timestampEnd & TEXT & YYYYMMDDHHMM & ISO timestamp end of averaging period - short format \\
\hline timestampStart & TEXT & YYYYMMDDHHMM & ISO timestamp start of averaging period - short format \\
\hline $\begin{array}{l}\text { swcFMDS1_deg } \\
\text { C }\end{array}$ & REAL & percent & Soil water content, gapfilled with MDS (numeric index I"\#I" increases with the depth, 1 is shallowest) \\
\hline swcFMDS1_qc & INTEGER & adimensional & Quality flag for tsFMDS\#. 0 = measured; 1 = good quality gapfill; 2 = medium; 3 = poor. \\
\hline $\begin{array}{l}\text { swcFMDS2_deg } \\
\text { C }\end{array}$ & REAL & percent & Soil water content, gapfilled with MDS (numeric index I"\#I" increases with the depth, 1 is shallowest) \\
\hline swcFMDS2_qc & INTEGER & adimensional & Quality flag for tsFMDS\#. 0 = measured; 1 = good quality gapfill; 2 = medium; 3 = poor. \\
\hline $\begin{array}{l}\text { swcFMDS3_deg } \\
\text { C }\end{array}$ & REAL & percent & Soil water content, gapfilled with MDS (numeric index I"\#" increases with the depth, 1 is shallowest) \\
\hline swcFMDS3_qc & INTEGER & adimensional & Quality flag for tsFMDS\#. 0 = measured; 1 = good quality gapfill; 2 = medium; 3 = poor. \\
\hline $\begin{array}{l}\text { swcFMDS4_deg } \\
\text { C }\end{array}$ & REAL & percent & Soil water content, gapfilled with MDS (numeric index I"\#I" increases with the depth, 1 is shallowest) \\
\hline swcFMDS4_qc & INTEGER & adimensional & Quality flag for tsFMDS\#. 0 = measured; 1 = good quality gapfill; 2 = medium; 3 = poor. \\
\hline $\begin{array}{l}\text { swcFMDS5_deg } \\
\text { C }\end{array}$ & REAL & percent & Soil water content, gapfilled with MDS (numeric index I"\#I" increases with the depth, 1 is shallowest) \\
\hline
\end{tabular}




\begin{tabular}{|c|c|c|c|}
\hline swcFMDS5_qc & INTEGER & adimensional & Quality flag for tsFMDS\#. 0 = measured; 1 = good quality gapfill; 2 = medium; $3=$ poor. \\
\hline tsFMDS1_degC & REAL & degree Celsius & Soil temperature, gapfilled with MDS (numeric index I"\#I" increases with the depth, 1 is shallowest) \\
\hline tsFMDS1_qc & INTEGER & adimensional & Quality flag for tsFMDS\#. 0 = measured; 1 = good quality gapfill; 2 = medium; 3 = poor. \\
\hline tsFMDS2_degC & REAL & degree Celsius & Soil temperature, gapfilled with MDS (numeric index l"\#l" increases with the depth, 1 is shallowest) \\
\hline tsFMDS2_qc & INTEGER & adimensional & Quality flag for tsFMDS\#. 0 = measured; 1 = good quality gapfill; 2 = medium; $3=$ poor. \\
\hline tsFMDS3_degC & REAL & degree Celsius & Soil temperature, gapfilled with MDS (numeric index I"\#I" increases with the depth, 1 is shallowest) \\
\hline tsFMDS3_qc & INTEGER & adimensional & Quality flag for tsFMDS\#. 0 = measured; 1 = good quality gapfill; $2=$ medium; 3 = poor. \\
\hline tsFMDS4_degC & REAL & degree Celsius & Soil temperature, gapfilled with MDS (numeric index I"\#|" increases with the depth, 1 is shallowest) \\
\hline tsFMDS4_qc & INTEGER & adimensional & Quality flag for tsFMDS\#. 0 = measured; 1 = good quality gapfill; 2 = medium; $3=$ poor. \\
\hline tsFMDS5_degC & REAL & degree Celsius & Soil temperature, gapfilled with MDS (numeric index I"\#|" increases with the depth, 1 is shallowest) \\
\hline tsFMDS5_qc & INTEGER & adimensional & Quality flag for tsFMDS\#. 0 = measured; 1 = good quality gapfill; 2 = medium; 3 = poor. \\
\hline
\end{tabular}




\section{Text SOM 1 Reconstruction of the stand development at Sorø (SOR-DK)}

The objective of this section is to describe how we reconstructed the stand development from the available data (Table SOM14), where exact data on thinning were not available. Therefore we used the yield table to fill the missing information assuming that management at the site was following the general recommendations for this site (Møller 1933). Because of considerable differences in tree densities, we needed to scale the management cycle of Møller (1933) to the empirical data from our stand. We used mainly Møller’s thinning schedule, i.e. at which age the stand was usually thinned. For this we reconstructed the stand backward, i.e. starting at 288 trees ha $^{-1}$ at age 90 yr in 2010 until 1921 . This tree density $(r)$, i.e. numbers of trees per ha, was increased in years when the schedule $(S)$ is one, i.e. forcing a thinning event. The degree of thinning is described by the two right hand side terms as a root function depending on the tree density itself. This procedure forced the fitting procedure to stay closely to the measured data at the end of the stand development. The fitted values for the empirical parameters $a$ and $c$ were $0.04128 \pm$ $0.01551(\mathrm{p}=0.044755 *$ ) and $-1.19594 \pm 0.13255(\mathrm{p}=0.000279 * * *)$, respectively. The results are displayed in Figure SOM2. Comparison with data after Møller's yield table shows that the reduction of tree density started earlier at Sorø than in the normal stand development. One probable reason for this is that the beech forest at Sorø was initiated underneath a canopy of a few remaining old beeches. This could have fostered more intensive self-thinning than under normal conditions. On the other hand the assumed initial number of beech trees of 6000 per ha is an overestimation. The tree density values in the first 20 years are very uncertain. On the other hand we are confident that the thinning scheme of Møller has been adopted by the Danish forestry management at least over a large part of the stand lifetime.

Table SOM14: New version on Management Information available

\begin{tabular}{|l|l|l|l|}
\hline Year/Periods & $\begin{array}{l}\text { Tree density } \\
{\left[\mathbf{h a}^{-1}\right]}\end{array}$ & Source & Method \\
\hline $1921-1930$ & 6000 & Møller (1933) + Th Kaspersens notes (assumed) & from yield table + notes from forester \\
\hline 1933 & 3550 & from Munds Målebog & assumed measured in $468 \mathrm{~m}^{2}$ \\
\hline 1944 & 968 & Taksationsbog & From volume and average DBH and height \\
\hline 1977 & 384 & Estimated by Sorø Akademi & from yield table \\
\hline 1992 & 369 & Estimated by Sorø Akademi & from yield table \\
\hline
\end{tabular}




\begin{tabular}{|l|l|l|l|}
\hline 1995 & 361 & Estimated by Sorø Akademi & from yield table \\
\hline $2005(03.2006)$ & 326.3 & DTU measurements (P87) & measured \\
\hline 2007 & 288 & DTU measurements (P87)[ANIB1] & measured \\
\hline
\end{tabular}

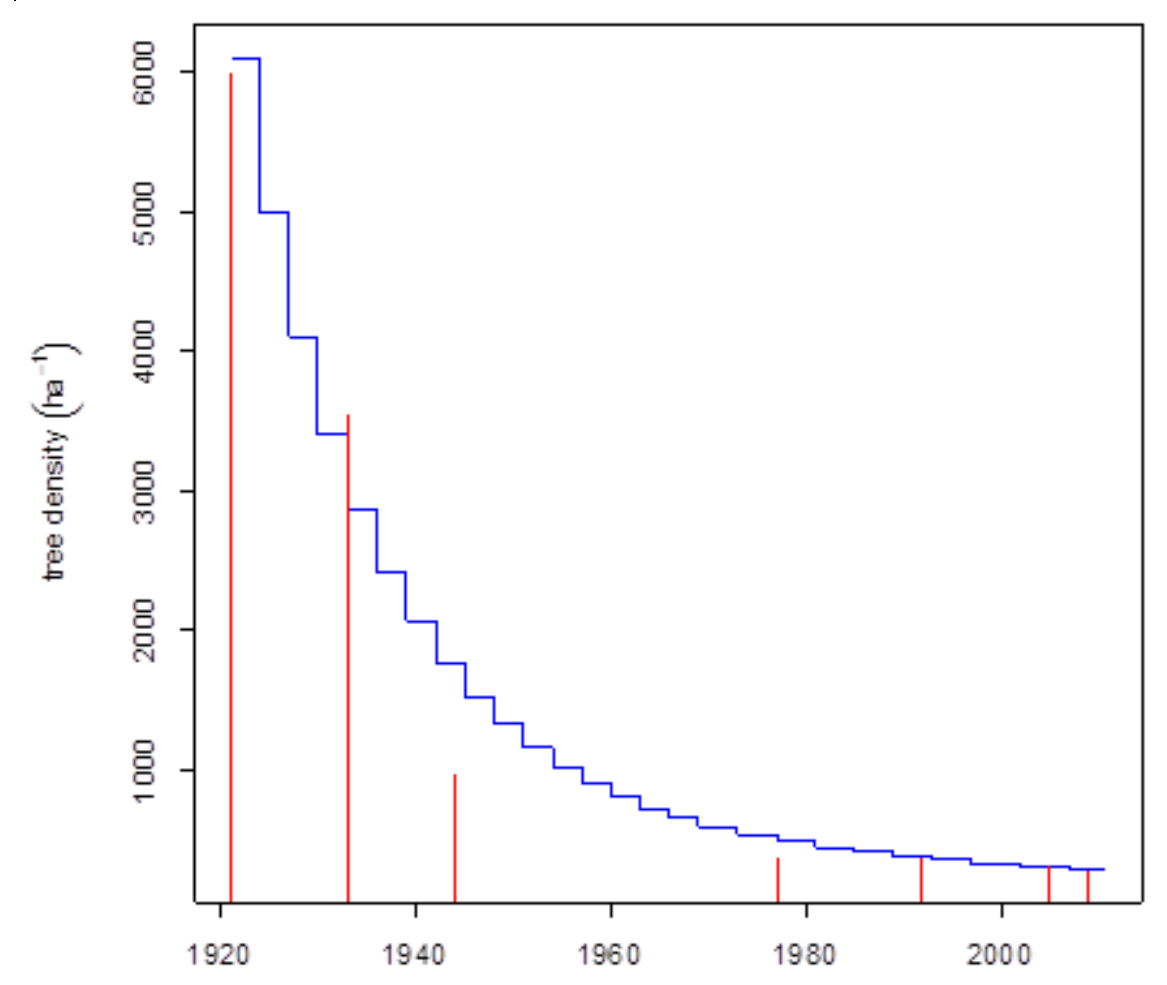

Figure SOM2. Reconstruction of the tree density at Sorø, division 335. During the years from 1921 to, at least, 1944 there was a canopy of old trees. The blue step function is the reconstructed series using the thinning intervals as in Møller (1933) and thinning intensities that match the observations best (red bars, see Table SOM14). 


\section{Thinning}

Unfortunately thinning of the trees is not anymore documented. Anders Grube, the current forest manager, explained the rule for thinning, which will be applied from now $(=2007+)$ on, is thinning interval = tree age / 10 in years. This rule of thumb indicates a slight change from Møller's suggestion, i.e. thinning every 5 years at this stand age. Only a very few examples on how much wood volume was actually thinned can be found. In one example, 2007, thinning was performed in division 336 (planted in 1941, i.e. 66 years) the standing stock was $999 \mathrm{~m}^{3}$ and he removed $40 \mathrm{~m}^{3}$, which is 999/4.11 $=243 \mathrm{~m}^{3} \mathrm{ha}^{-1}$ and 40/4.11 $=10 \mathrm{~m}^{3} \mathrm{ha}^{-1}$ for standing stock and harvested wood, respectively. This means fro this particular site a relative extraction of $4 \%$. This is smaller than Møller's suggestion (ca. $11 \%$, for this age, Møller (1933)). Measurements of tree growth as part of contemporary forest planning (by Klaus Wunsch (KW-plan, c/o) are only performed on some divisions, none of them in the fetch of the tower. For this reason we decided to use the available data from the forest owner, Sorø Akademi (Table SOM14), and use yield table information and tree rings to reconstruct thinning events and the stand development. We concentrate on division 335 where the inventory, ecological and meteorological measurements have been taken. With this approach it is not possible to reconstruct the exact thinning activity in a certain year, but instead the general forest development is being reconstructed. The thinning events are only accurate within a 3-5 years period.

\section{References}

Møller, C. M. (1933). "Bonitetstabeller og bonitetsvise tilvækstoversigter for bog, eg og rødgran i Danmark." Dansk Skovforenings Tidskrift 18: $457-513,537-623$. 